\title{
Molecular Biological Tools in Cancer Diagnostics with a Case Study
}

\author{
Fawzaan Hashmi
}

Mankato West High School

\begin{abstract}
Projects of cancer diagnostics are significantly variegated. Manifestly, however, understanding molecular biology can accomplish a great deal in the midst of any diagnostic strategy. This report will seek to indicate some of the key bases of molecular biomedical study, including the foundations of cell growth, cell death, and genetics, together with specific instances of protein classification, as they pertain to cancer. Methodologies of cancer analysis and treatment that cover similar molecular ground will then be elucidated. This will all serve to anchor an examination of the MET/phosphoinositide 3-kinase $(\mathrm{PI} 3 \mathrm{~K}) /$ protein kinase B $(\mathrm{AKT}) /$ mechanistic target of rapamycin (mTOR) pathway, linked to problematic cell proliferation in on cogenesis.
\end{abstract}

Keywords: Cancer, Metastasis, PI3k/AKT/mTor, Kinase, Ubiquitin, Angiogenesis, HER2.

\section{Introduction}

\section{Cell Growth}

A normal cell has relatively equivalent copies of each gene. But a cancer cell has a lot in one area and none in another, for example. DNA fingerprinting is something brought up with no real significance. MPF is made of Cyclin/Cyclin Dependent Kinase (CDK); CyclinB/CDK1 is a specific type that deals with the Mphase checkpoint. The G1/S checkpoint is the point of no return, so it functions as a restriction point (Cooper, 1970). In contrast, the G0 phase is quiescent i.e. inactive or dormant. The CyclinB/CDK1 protein kinase complex is also key to the beginning of mitosis by triggering the disassembly of nuclear pores and the nuclear membrane and lamina (allowing the spindle to interface with condensed chromosomes) (Spring et al., 2016).

Retinoblastoma, the disease and the gene, is abbreviated as RB. Usually it refers to a condition involving tumors in the eyes of children. The protein for RB inhibits the cell cycle between the G1 and $S$ phases. CyclinD/CDK4 phosphorylates $\mathrm{Rb}$ and activates the E2F transcription factor during G1, helping move the cell to the $\mathrm{S}$ phase. The regulation of CDKs and thus of cell growth can be enforced by Cyclins, phosphorylation, and CDK inhibitors. P53 induces p21, which is a CDK inhibitor.

\section{Cell Death}

1. Necrosis is unintentional cell death (Linkerman and Green, 2014).

2. Apoptosis is purposeful, programmed cell death or PCD. This encompasses cell shrinking, membrane blebbing, apoptotic bodies, and phagocytosis.

3. Necroptosis (programmed necrosis) and pyroptosis are intermediaries of both inflammatory cell death and apoptosis (Man et al., 2017).

4. Autophagy is self eating. Sometimes autophagy helps treat cancer, as cancer requires nutrients.

Caspases cause protease activation, which is crucial for apoptosis. Initiators activate the executioners, like caspase-3, a zymogen or proenzyme (a not-yet-activated enzyme), which cut the cytoskeleton (Walters et al., 2009). Caenorhabditis elegans is a $1 \mathrm{~mm}$ thick roundworm in which every cell, and the cell fate, is known and understood; it has been used to aid in developing our knowledge of the genes and inhibitors of apoptosis. Intrinsic apoptosis can be initiated by DNA damage, while extrinsic apoptosis deals with death ligands and death receptors (a programmed operation crucial to the formation of, for instance, webbed hands). Death domains activate caspases which leads to apoptosis and inflammation. The tumor necrosis 
factor receptor type 1-associated death domain protein is encoded by the TRADD gene. FAS is an apoptosis receptor. And apoptotic protease activating factor 1 (apaf-1) forms a central hub for apoptosis. The apoptosome is a large quaternary protein structure formed during apoptosis. The Bcl-2 family regulates apoptosis in the mitochondria (Soria and Gauthier, 2013).

A fusion protein consists of multiple domains; anti-PD-1 is pembrolizumab, which boosts the immune system in order to inhibit the tumor (Sehgal, 2015). Killer T cells, along with CD8, APC, and PD, lead to programmed cell death. Cancer cells stop the T-cell from killing them by expressing PD1-L. If every tumor cell expresses this, then the cancer will continue to thrive. Small molecule inhibitors are chemicals that are taken as oral pills to fight cancer; nylotarg is an antibody that goes after CD33. Phosphate buffered saline (PBS) is $0.9 \% \mathrm{Na}$. Chemiluminescence is the emission of light during a chemical reaction that does not produce large amounts of heat.

\section{Molecular Genetics}

Annealing is hybridizing, the pairing of complementary nucleic acid sequences. A primer is a single stranded oligonucleotide with a denaturing temperature of 94-100 degrees celsius. The procedure entails that the experimenter anneals primers to the part of the gene that is to be amplified and cools to 55-60 degrees celsius. The polymerizing temperature is 70-72 degrees celsius. Reverse transcriptase polymerase chain reaction (RT-PCR) is used for amplifying mRNA (Rio, 2014). Because RNA is very degradable, there are many more active RNAses. Real time PCR is commonly designated as Quantitative PCR (qPCR). And Quantitative Quantitative Reverse Transcription PCR (qRt-PCR) describes the process whereby a given RNA sequence leads to complementary DNA (cDNA) and the PCR is observed quantitatively (Overbergh et al., 2003).

Non-coding RNA (ncRNA) is not translated to protein, and these non-coding fragments can be long, short, ribosomal, or transfer RNAs (Diamantopoulos et al., 2018). Splicing using small nuclear RNA (snRNA) increases variability; this is why introns exist, in addition to the use of enforcers (Frías-Lasserre and Villagra, 2017). Branch points and consensus sequences are included in introns and allow for the stability of its molecular structure and the molecular structure of the post-excision nucleic acid. This occurs with the help of a spliceosome; snRPs help form spliceosomes, along with other components. A lariat is a ring of intron segments that has been spliced out of a messenger ribonucleic acid molecule by enzymes.

Alternative splicing allows a single gene to code for multiple proteins. For example, BimL/El proteins protect BimS proteins from apoptosis. The control of alternative splicing is not well known. Single nucleotide polymorphisms (SNPs) are single nucleotide substitutions in a genome; they are crucial in studying specific "cohorts" with regard to genomic regions. Alternative promoters allow genes to have multiple transcripts. Apolipoprotein B (apo B) gene is a gene where the mRNA can be edited, through cytidine deamination in the small intestine (Davidson, 1994). Inheritance

$\mathrm{RB}$, to return, is familial and inherited, which is particularly harmful. It is also a bilateral disease, so it is present in both eyes. Sporadic, not-inherited, abnormalities occur much later, and only in one eye. LOH is the loss of heterozygosity.

\section{Relevant Protein Roles}

DNA has no real, concrete function, and RNA has little use, but protein is an extremely operational set of molecules. To wit, there are 20 thousand genes but millions of proteins (the proteome). The transactivation domain is a transcription factor scaffolding domain, where proteins act upon genes.

P53 needs to add phosphate groups to 24 spots, so there are $2^{\wedge} 24$ possibilities. Fos 1,2 is a proto-oncogene; oncogenes are genes or proteins that cause cancer; although this term refers to the gene and the protein, the protein is what actually causes cancer. JunA, B, and C are proteins that form the AP-1 early response transcription factor when combined with Fos (Curran and Franza et al., 1988).

Phosphatases dephosphorylate, and proteases are molecular scissors. Ligases connect elements together, while transferases move them in accordance with signal pathways. DNAses/RNases are enzymes that cleave DNA and RNA, respectively. Helicases "unzip" double stranded chains. Chaperone proteins help proteins fold. Secondary structure involves more than just beta pleated sheets and alpha helices; it has random coils with no specific structure. 
X-Ray crystallography can be used to determine the molecular structure of crystalline structures. Serine, threonine, and tyrosine have hydroxyl groups for the transfer of phosphate groups from ATP, a reversible process. Kinases tend to be oncogenes, activating proteins which are associated with cancer; when a kinase is always on, proliferation that leads to cancer occurs. Relatedly, c-Src is a proto-oncogene tyrosine-protein kinase. Their opposite, phosphatases, are tumor suppressor genes (TSGs), which, naturally, implies that phosphatase is a cancer inhibitor. TSGs regulate the cell cycle and promote apoptosis. They are recessive, so both copies must be lost in order to stop suppressing cancer (Wang et al., 2013). P16 is another example of a TSG.

Ubiquitin is a protein of 8.5 kilodalton $(\mathrm{kDa}) .1$ dalton is a unit of weight that is $1 / 12$ the weight of a carbon, which is $1.67 \times 10^{\wedge}-27 \mathrm{~kg}$. Ubiquitin is put on lysine residues.

Ubiquitin tag leads the protein to the proteasome, where it will get degraded (Callis, 2014). Peptidase breaks peptide bonds to yield individual amino acids, which are then degraded and recycled in the cytoplasm.

Steps for proteins to get ubiquitin:

E1 - Ubiquitin is activated by binding to the protein, which requires ATP of which there are two types

$\mathrm{E} 2$ - This is the enzyme that puts ubiquitin on the substrate and takes it to the E3. There are 60 types.

E3 - In E3, it binds to the substrate, recognizes it, and targets it with drugs. There are 600-800 types

E4 - In E4, Ubiquitin is removed (Myung et al., 2001).

The ubiquitin proteasome system is denoted as the UPS. Proteins need not always have something problematic with them in order to be recycled.

\section{P53}

P53 is a protein of 53kDa. It is a transcription factor that controls the cell cycle and helps with DNA repair, generating new cells with too many mutations to commit apoptosis. It can be described as the guardian of the genome (Allton et al., 2009). The necessity of repair via the cell cycle is fulfilled by the MDM2 oncogene. The Mdm2 protein operates as an E3 ubiquitin ligase that degrades p53. Appositely, nutlin prevents p53 from getting ubiquitinated. P53 knockout mouse has proven to be useful to study in-vivo and in-vitro contexts (Shi and $\mathrm{Gu}, 2012$ ). Kaplan-Meier graphs measure survival, with time on the $\mathrm{x}$ and percent survival on the $y$. It was indicated that mice were dying when they were heterozygous for the p53 gene, as p53 binds as a tetramer, not a dimer. P53 has no deletions that alter it but only point mutations. P53 targets p21, a CDK inhibitor (Muller et al., 2011). More target genes are apoptotic, like PUMA or Noxa. The XPC protein is important for nucleotide excision repair, and promotes MDM2-directed p53 degradation.

\section{Molecular Biology and Angiogenesis}

Hemangioblasts form blood islands in the yolk, and vasculogenesis is when new blood vessels form that were not already there. Conversely, angiogenesis is when new blood vessels from from previous blood vessels and arteriogenesis is the development of arteries and veins from smaller vessels. Lymphangiogenesis is the development of lymph vessels. The postcapillary venule is the locale where intravasation and extravasation occur, during slower speeds of flow. Hypoxia is a potent inducer of angiogenesis. It is usually $.5-1 \%$ oxygen in laboratory environments, while normoxia is around $20 \%$ oxygen.

The link between angiogenesis and cancer was discovered by Dr. Judah Folkman, who developed the first atrioventricular implantable pacemaker. Growth factors can induce angiogenesis, which take place on endothelial cells. Platelet-derived growth factors (PDGFs) bind to vascular endothelial growth factor (VEGF) receptors on epithelial cells. Vascular permeability and leakage relates to the VEGF; if there is too much VEGF from proliferation, blood vessels form too quickly and leak (Duffy et al., 1970). VEGF is part of a family of proteins, with the most common besides VEGF or VEGF-A being VEGF 165 (lacking exon 6). At all times there are both pro and anti angiogenesis factors present. Anti-angiogenesis factors are angiostatin and endostatin. Monoclonal antibodies can be used to stop angiogenesis (Padma, 2015). Thalidomide can provide pregnant women morning sickness relief, but has caused cancer in the past. Avastin is an anti-VEGF antibody, a humanized monoclonal antibody. Avastin needs to be taken throughout 
an entire lifespan; bevacizumab is another name for avastin. E3 ubiquitin ligase transfers ubiquitin from the E2 to the protein substrate. HIF1alpha is the master transcriptional regulator of cellular and developmental reactions to hypoxia. Prolyl 4 hydroxylase catalyzes the incorporation of oxygen into organic substances.

\section{Cancer Diagnostics}

E-cadherin is responsible for metastasis. Metastasis causes $90 \%$ of cancer deaths and perhaps even more (Tang et al., 2018). If the primary tumor is very big, then there is much less competition elsewhere. This is another multistep process: only $.01 \%$ of cells survive metastasis. 1 million cells metastasize in cancer; most are killed. Primary tumors are where tumors originate from and secondary tumors are where the tumor metastasizes. $1 \mathrm{~mm}$ in diameter, the tumor capillaries can't access the middle, so blood vessels will grow into the tumor as the tumor grows. Next, angiogenesis starts and, suddenly, the entire body is available to cancer cells via nearby blood vessels. Cancer cells can't get into arteries or veins, which are large and firm, so they enter via capillaries and venules. The basement membrane is a thin layer of protein fibers that separates the epithelium from the underlying tissue. Intravasation is the invasion of cancer cells through the basement membrane into blood or lymphatic vessels. And in turn, extravasation is the leakage of mostly blood and lymph into the surrounding tissue. Bioinformatics is a field that develops methods to understand biological data and can be applied to data-based investigations of cancer diagnostics.

The seed and soil model posits that cancer cells are akin to seeds and that organs resemble soil under the correct conditions. However, this also depends on circulation, with the amount of blood travelling to the lungs functioning as an example. Relapse is when cancer cells survive the treatment and reappear later on. Tumor cells can be dormant for five years. The only way the immune system would kill cancer cells is if a mutation occurs in the antigens.

Nucleoside analogs are anticancer and antiviral drugs (Mikhailopulo and Miroshnikov, 2010). Dry ice can be used to preserve hair during chemotherapy. Oncolytic viruses kill and infect cancer cells. Hepatocellular carcinoma or HCC is the most common type of liver cancer. Reticulocytes are immature red blood cells that can help determine how many new red blood cells are being made.

Targeted therapies are drugs that block the growth and spread of cancer. They achieve this by interfering with specific molecules involved in tumor growth. Chemotherapy and radiation target proliferating cells. However, this is not ideal, because some cells proliferate but are not cancerous.

Treatment for different stages of cancer:

Stage 1- Surgery.

Stage 2- Surgery, chemotherapy, and radiation.

Stage 3- Surgery, chemotherapy, and radiation.

Stage 4- Chemotherapy and radiation.

Cancer Treatment Typology (Carcinoma):

1. Surgery.

2. Chemotherapy. If 5 fluoro-uracil (5FU) is used, then some proliferating cells in the GI tract and bone marrow will be affected. Anemia and a compromised immune system will sprout as a result.

3. Radiation. High energy molecules from radiation damage the DNA in the cancerous cells. However, the shadow will still affect normal cells, albeit with less intensity.

4. Target therapies. Small molecule inhibitors (SMIs) are productive because they can be ingested orally. The molecule binds to a growth factor and prevents binding.

\section{ABCDE framework for melanoma invasion}

Asymmetrical, border, color, diameter, and evolution. Teratoma is a germ cell tumor involving multiple tissues that develops in fetuses. Epithelial-mesenchymal transition or EMT designates the process whereby an epithelial cell morphs into a mesenchymal cell. Ultimately, this leads to a different phenotype (specifically, a change in morphology and gene expression). Epithelial cells have cytokeratin, whereas mesenchymal cells have vimentin and an E-cadherin decrease. Here, epithelial cells are stationary, whereas 
mesenchymal cells are motile. This a common characteristic of all carcinomas. There are 3 types of EMT: development, wound healing, and cancer (Kalluri and Weinberg, 2009). The opposite of EMT is MET, which refers to the mesenchymal-epithelial transition. With regards to calcium, two E-cadherins connect two epithelial cells. In special stains, the nucleus is usually identified by a blue hue. DAPI is a fluorescent stain that binds strongly to adenine-thymine rich regions in DNA, and as such it is heavily used in fluorescence microscopy. Endocytosis is increased when an antibody is attached.

\section{HER2}

Crosstalk is when two signaling pathways interact (Vert and Chory, 2011). The hormone therapy for breast, prostate, testicular, and cervical cancer is anti-estrogens or anti-androgens. Herceptin is a part of the epidermal growth factor (EGF) receptor family; it treats HER2 (human epidermal growth factor receptor 2) positive breast cancer (trastuzumab was its original, scientific, name). Cancer cells have 1-2 million HER2s whereas normal cells have an HER2 count of 10,000. In this scenario, there is a link between HER2+ and cancer. HER2 surpluses manifest when there is too much protein. This must also mean that there is too much mRNA and DNA. HER2+ takes herceptin and does not hurt normal cells. Cardiac muscle cells may overexpress HER2, so it is harmful if someone takes herceptin when they are not HER2+ (Iqbal and Iqbal, 2014).

The same gene can be copied many times over on a single chromosome, even to the point where 20 genes could exist on one chromosome when only one should. It is possible to screen people at the DNA level by using fluorescent in situ hybridization (FISH).

\section{Clinical Trials}

Phase 1- A small study, just starting to put 10-20 people. Here, aspects of safety, such as mortality and side effects, are being tested.

Phase 2- This phase consists of 100-200 people, with the goal of testing efficacy.

Phase 3- There are 1000-3000 people imbricated. There are people from multiple hospitals, testing success.

The Kaplan-Meier curve is used to plot drugs. The standard of care is the level of care a medical provider should provide to his patients. Cancer studies do not contain placebos.

\section{The PI3K/AKT Pathway: Looking at A549 cancer cells and the Expression of GRP78}

\section{MET}

Met or C-MET is a tyrosine kinase and hepatocyte growth factor receptor (HGFR) protein that is responsible for tumor growth. Angiogenesis, along with processes such as mitogenesis, morphogenesis, EMT, gastrulation, and myoblast migration, mediates invasive growth (Costa et al., 2018). The MET pathway allows for scatter, which is where certain cells dissociate from each other due to metalloprotease production. This often leads to metastasis. We are dealing with a proto-oncogene.

The PI3K/AKT pathway relates to quiescence and proliferation (and, of course, cancer) ("Explore $\mathrm{PI} 3 \mathrm{~K} / \mathrm{AKT}$ "). It generates apoptosis, and promotes proliferation and differentiation in neural stem cells (Rafalski and Brunet, 2011). The phosphatase and tensin homolog (PTEN) and cAMP response elementbinding (CREB) proteins are, in part, closely related. PTEN acts as the TSG, whereas CREB is a transcription factor for a process that leads to proliferation. Finally, long term potentiation is a process that only occurs when the PI3K/AKT pathway is active.

\section{A549}

A549 cells are adenocarcinomic human alveolar basal epithelial cells linked to lung cancer. They are conducive to sustained analysis and study and important for the diffusion of water. Paclitaxel, docetaxel, and bevacizumab are chemotherapeutic drugs that have been heavily tested on A549 cells.

GRP78 is also known as binding immunoglobulin protein (BiP), heat shock 70 kilodalton protein 5 
(HS70kDap5), Byun1, and HSPA5. It is a chaperone protein located in the endoplasmic reticulum lumen that binds proteins shortly after synthesis. Afterwards, GRP78 preserves these proteins in a state ripe for folding and oligomerization. The unfolded protein response (UPR) can then be seen as indicative of the tumor microenvironment, as it triggers a cancer stress response and the master UPR regulator. Further, this engenders chemoresistance.

\section{$H G F$}

Hepatocyte Growth Factor (HGF) provides many clinical applications. HGF, which is a hepatocyte mitogen, is most notable for its role in epithelial cell proliferation. HGF is also responsible for curtailing organ disease, which suggests a possibility that HGF can be therapeutic against pathologies alike (Johnson et al., 1993). It pertains to scatter factor (SF) and is derived from cancer associated fibroblast (CAF). The paracrine nature is crucial in terms of motility and morphology (Woo, 2015). For instance, the proceeding of myogenesis, EMT, and angiogenesis demonstrate this utility of HGF. HGF also acts as a cytokine toward epithelial cells. The relationship between the MET/PI3K/AKT pathway and GRP78 is then made apparent. CAF tumor microenvironments is the context for this analysis of chemoresistant cancer.

A microfluidic microenvironment might be an ideal platform. It's abilities of actuation, bioparticle manipulation, efficient, accurate mass delivery, and adaptation to diverse tumors are valuable.

\section{$C A F, H G F$, and $M E T / P I 3 K / A K T$}

When HGF binds to MET, the tyrosine kinases are phosphorylated i.e. activated (Liu et al., 2017). GRP78 upregulation is caused by CAF derived HGF binding to the MET/PI3K/Akt/mTOR pathway (Deying et al., 2017). Paclitaxel increases cell apoptosis; inhibiting PI3K or GRP78 allows for chemoresistance to end and thus allows chemotherapy to proceed with increased efficacy (Ying et al., 2015).

\section{Conclusion}

Further study of molecular biology would illustrate more tactics of molecular manipulation related to cancer diagnostics and therapy. The chemotherapy strategy highlighted here presumes a region of molecular interactions, namely the MET/PI3K/Akt/mTOR pathway but also a wide range of biomolecular processes connected to cell proliferation and oncogenesis. In this way, the given chemotherapy strategy and its grounding in a molecular analysis encourages the emphasis on the study of molecular biology in cancer diagnostics advocated above.

\section{Works Cited}

[1.] Allton, Kendra, et al. "Trim24 Targets Endogenous p53 for Degradation." Proceedings of the National Academy of Sciences of the United States of America, National Academy of Sciences, 14 July 2009, www.ncbi.nlm.nih.gov/pubmed/19556538.

[2.] Callis, Judy. "The Ubiquitination Machinery of the Ubiquitin System." The Arabidopsis Book, American Society of Plant Biologists, 6 Oct. 2014, www.ncbi.nlm.nih.gov/pmc/articles/PMC4196676/.

[3.] Cooper, Geoffrey M. "Regulators of Cell Cycle Progression." The Cell: A Molecular Approach. 2nd Edition., U.S. National Library of Medicine, 1 Jan. 1970, www.ncbi.nlm.nih.gov/books/NBK9962/.

[4.] Costa, Ricardo L. B., et al. "Targeting the PI3K/AKT/MTOR Pathway in Triple-Negative Breast Cancer: a Review." SpringerLink, Springer US, 7 Feb. 2018, link.springer.com/article/10.1007\%2Fs10549-018-4697-y.

[5.] Curran, T, and B R Franza. "Fos and Jun: the AP-1 Connection." Cell, U.S. National Library of Medicine, 4 Nov. 1988, www.ncbi.nlm.nih.gov/pubmed/3141060.

[6.] Davidson, N O. "RNA Editing of the Apolipoprotein B Gene A Mechanism to Regulate the Atherogenic Potential of Intestinal Lipoproteins?" Trends in Cardiovascular Medicine, U.S. National Library of Medicine, 1994, www.ncbi.nlm.nih.gov/pubmed/21244872.

[7.] Deying, Wei, et al. "CAF-Derived HGF Promotes Cell Proliferation and Drug Resistance by upRegulating the c-Met/PI3K/Akt and GRP78 Signalling in Ovarian Cancer Cells." Bioscience Reports, Portland Press Ltd., 10 Apr. 2017, www.ncbi.nlm.nih.gov/pmc/articles/PMC5469328/.

[8.] Diamantopoulos, Marios A, et al. "Non-Coding RNAs: the Riddle of the Transcriptome and Their 
Perspectives in Cancer." Annals of Translational Medicine, AME Publishing Company, June 2018, www.ncbi.nlm.nih.gov/pmc/articles/PMC6046292/.

[9.] Duffy, Angela M. "Vascular Endothelial Growth Factor (VEGF) and Its Role in Non-Endothelial Cells: Autocrine Signalling by VEGF." Madame Curie Bioscience Database [Internet]., U.S. National Library of Medicine, 1 Jan. 1970, www.ncbi.nlm.nih.gov/books/NBK6482/.

[10.] "Explore PI3K/AKT." BioOncology, www.biooncology.com/pathways/cancer-tumortargets/pi3k.html.

[11.] Johnson, Mark, et al. "Hepatocyte Growth Factor Induces Proliferation and Morphogenesis in Nonparenchymal Epithelial Liver Cells.” Hepatology, vol. 17, no. 6, 1993, pp. 1052-1061., doi:10.1002/hep.1840170618.

[12.] Frías-Lasserre, Daniel, and Cristian A Villagra. "The Importance of NcRNAs as Epigenetic Mechanisms in Phenotypic Variation and Organic Evolution." Frontiers in Microbiology, Frontiers Media S.A., 22 Dec. 2017, www.ncbi.nlm.nih.gov/pmc/articles/PMC5744636/.

[13.] Iqbal, Nida, and Naveed Iqbal. "Human Epidermal Growth Factor Receptor 2 (HER2) in Cancers: Overexpression and Therapeutic Implications." Molecular Biology International, Hindawi Publishing Corporation, 2014, www.ncbi.nlm.nih.gov/pmc/articles/PMC4170925/.

[14.] Kalluri, Raghu, and Robert A Weinberg. "The Basics of Epithelial-Mesenchymal Transition." The Journal of Clinical Investigation, American Society for Clinical Investigation, June 2009, www.ncbi.nlm.nih.gov/pmc/articles/PMC2689101/.

[15.] Linkermann, Andreas, and Douglas R Green. "Necroptosis." The New England Journal of Medicine, U.S. National Library of Medicine, 30 Jan. 2014, www.ncbi.nlm.nih.gov/pmc/articles/PMC4035222/.

[16.] Liu, Fang, et al. "HGF Induces EMT in Non-Small-Cell Lung Cancer through the HBVR Pathway." European Journal of Pharmacology, U.S. National Library of Medicine, 15 Sept. 2017, www.ncbi.nlm.nih.gov/pubmed/28554854.

[17.] Man, Si Ming, et al. "Molecular Mechanisms and Functions of Pyroptosis, Inflammatory Caspases and Inflammasomes in Infectious Diseases." Immunological Reviews, U.S. National Library of Medicine, May 2017, www.ncbi.nlm.nih.gov/pmc/articles/PMC5416822/.

[18.] Mikhailopulo, I A, and A I Miroshnikov. "New Trends in Nucleoside Biotechnology." Acta Naturae, A.I. Gordeyev, July 2010, www.ncbi.nlm.nih.gov/pmc/articles/PMC3347554/.

[19.] Muller, Patricia A J, et al. "p53 And Its Mutants in Tumor Cell Migration and Invasion." The Journal of Cell Biology, The Rockefeller University Press, 24 Jan. 2011, www.ncbi.nlm.nih.gov/pubmed/21263025.

[20.] Myung, J, et al. "The Ubiquitin-Proteasome Pathway and Proteasome Inhibitors." Medicinal Research Reviews, U.S. National Library of Medicine, July 2001, www.ncbi.nlm.nih.gov/pmc/articles/PMC2556558/.

[21.] Overbergh, L, et al. "The Use of Real-Time Reverse Transcriptase PCR for the Quantification of Cytokine Gene Expression." Journal of Biomolecular Techniques : JBT, The Association of Biomolecular Resource Facilities, Mar. 2003, www.ncbi.nlm.nih.gov/pmc/articles/PMC2279895/.

[22.] Padma, Viswanadha Vijaya. "An Overview of Targeted Cancer Therapy." BioMedicine, China Medical University, Nov. 2015, www.ncbi.nlm.nih.gov/pubmed/26613930.

[23.] Rafalski, Victoria A., and Anne Brunet. "Energy Metabolism in Adult Neural Stem Cell Fate." Progress in Neurobiology, vol. 93, no. 2, 2011, pp. 182-203., doi:10.1016/j.pneurobio.2010.10.007.

[24.] Rio, Donald C. "Reverse Transcription-Polymerase Chain Reaction." Cold Spring Harbor Protocols, U.S. National Library of Medicine, 3 Nov. 2014, www.ncbi.nlm.nih.gov/pubmed/25368309.

[25.] Sehgal, Alison, et al. "Programmed Death-1 Checkpoint Blockade in Acute Myeloid Leukemia." Expert Opinion on Biological Therapy, U.S. National Library of Medicine, 2015, www.ncbi.nlm.nih.gov/pmc/articles/PMC4778424/.

[26.] Shi, Dingding, and Wei Gu. "Dual Roles of MDM2 in the Regulation of p53: Ubiquitination Dependent and Ubiquitination Independent Mechanisms of MDM2 Repression of p53 Activity." Genes \& Cancer, SAGE Publications, Mar. 2012, 
www.ncbi.nlm.nih.gov/pmc/articles/PMC3494363/.

[27.] Soria, Bernat, and Benoit R Gauthier. "Dual Trade of Bcl-2 and Bcl-XL in Islet Physiology: Balancing Life and Death with Metabolism Secretion Coupling." Diabetes, American Diabetes Association, Jan. 2013, www.ncbi.nlm.nih.gov/pmc/articles/PMC3526041/.

[28.] Spring, Laura, et al. "Targeting the Cyclin D-Cyclin-Dependent Kinase (CDK) 4/6-Retinoblastoma Pathway with Selective CDK 4/6 Inhibitors in Hormone Receptor-Positive Breast Cancer: Rationale, Current Status, and Future Directions." Discovery Medicine, U.S. National Library of Medicine, Jan. 2016, www.ncbi.nlm.nih.gov/pubmed/26896604.

[29.] Tang, Maggie K S, et al. "Soluble E-Cadherin Promotes Tumor Angiogenesis and Localizes to Exosome Surface." Nature Communications, Nature Publishing Group UK, 11 June 2018, www.ncbi.nlm.nih.gov/pubmed/29891938.

[30.] Vert, Grégory, and Joanne Chory. "Crosstalk in Cellular Signaling: Background Noise or the Real Thing?" Developmental Cell, U.S. National Library of Medicine, 13 Dec. 2011, www.ncbi.nlm.nih.gov/pmc/articles/PMC3281494/.

[31.] Walters, Jad, et al. "A Constitutively Active and Uninhibitable Caspase-3 Zymogen Efficiently Induces Apoptosis." The Biochemical Journal, Portland Press Ltd., 10 Dec. 2009, www.ncbi.nlm.nih.gov/pubmed/19788411.

[32.] Wang, Haiyan, et al. "TSG: a New Algorithm for Binary and Multi-Class Cancer Classification and Informative Genes Selection." BMC Medical Genomics, BioMed Central, 2013, www.ncbi.nlm.nih.gov/pubmed/23445528.

[33.] Woo, Jong Kyu, et al. "Humanized Anti-Hepatocyte Growth Factor (HGF) Antibody Suppresses Innate Irinotecan (CPT-11) Resistance Induced by Fibroblast-Derived HGF." Oncotarget, Impact Journals LLC, 15 Sept. 2015, www.ncbi.nlm.nih.gov/pmc/articles/PMC4695169/.

[34.] Ying, Li, et al. "Cancer Associated Fibroblast-Derived Hepatocyte Growth Factor Inhibits the Paclitaxel-Induced Apoptosis of Lung Cancer A549 Cells by Up-Regulating the PI3K/Akt and GRP78 Signaling on a Microfluidic Platform." PLOS ONE, Public Library of Science, 26 June 2015, journals.plos.org/plosone/article?id=10.1371\%2Fjournal.pone.0129593. 